NBI-HE-93-75

December 1993

\title{
On sphaleron deformations induced by Yukawa interactions
}

\author{
Minos Axenides \\ The Niels Bohr Institute \\ University of Copenhagen, 17 Blegdamsvej, 2100 Copenhagen, Denmark \\ Andrei Johansen 2 \\ The St.Petersburg Nuclear Physics Institute \\ Gatchina, St.Petersburg District, 188350 Russia \\ Holger Bech Nielsen 9 \\ The Niels Bohr Institute \\ University of Copenhagen, 17 Blegdamsvej, 2100 Copenhagen, Denmark
}

\begin{abstract}
Due to the presence of the chiral anomaly sphalerons with Chern-Simons number a half $(\mathrm{CS}=1 / 2)$ are the only static configurations that allow for a fermion level crossing in the two-dimensional Abelian-Higgs model with massless fermions, i.e. in the absence of Yukawa interactions. In the presence of fermion-Higgs interactions we demonstrate the existence of zero energy solutions to the one-dimensional Dirac equation at deformed sphalerons with $\mathrm{CS} \neq 1 / 2$. Induced level crossing due to Yukawa interactions illustrates a nontrivial generalization of the Atiyah-Patodi-Singer index theorem and of the equivalence between parity anomaly in odd and the chiral anomaly in even dimensions. We discuss a subtle manifestation of this effect in the standard electroweak theory at finite temperatures.
\end{abstract}

\footnotetext{
${ }^{1}$ e-mail:axenides@nbivax.nbi.dk

${ }^{2}$ e-mail:johansen@lnpi.spb.su

${ }^{3}$ e-mail:hbech@nbivax.nbi.dk
} 


\section{Introduction}

The standard model of electroweak interactions violates baryon number through the chiral anomaly [i]. One of the reasons for this is the periodic structure of the vacuum in non-abelian gauge theories. The distinct ground states are labeled by integer values of $N_{C S}$, the Chern-Simons number of the $S U(2)$ gauge field $A_{\mu}$

$$
N_{C S}=\frac{g^{2}}{16 \pi^{2}} \int_{M^{3}} \operatorname{Tr}\left(A d A+\frac{2}{3} A^{3}\right)
$$

where $M^{3}$ is a 3-dimensional manifold.

The height of the potential barrier between adjacent vacua is given by the energy of the sphaleron $E_{s p}=O\left(M_{W} / \alpha\right)$ ( $\alpha$ is $S U(2)$ gauge coupling). It is a static and unstable solution to the classical equations of the $S U(2)$ theory. Any transition between vacuum sectors is accompanied by a net change in both baryon and lepton numbers due to level crossing

$$
\Delta B=\Delta L=n_{f} \Delta N_{C S}=\frac{n_{f}}{32 \pi^{2}} \int_{M^{4}} \operatorname{Tr} F^{2}=n_{f} \int_{M^{4}} \partial_{\mu} j_{5}^{\mu} \propto n_{f}\left(n_{+}-n_{-}\right),
$$

where $M^{4}=M^{3} \times S^{1}$ is a four dimensional euclidean manifold. Here $n_{f}$ is the number of families, $F=d A+A^{2}$ is the $S U(2)$ field strength and $n_{ \pm}$is the number of left(right)-handed zero modes of the 4-dimensional Dirac operator $i \not D_{4}(A)$. Such a transition is signaled by the presence of a zero energy normalizable solution to the 4-dimensional Dirac equation (quantum tunneling) or the 3-dimensional one in a sphaleron-like background (thermal jump).

The anomaly of the chiral current becomes an anomaly in the baryon and lepton currents because the standard model is chiral. At zero temperature and energy B-violating transitions are exponentially suppressed. At high temperature and in thermal equilibrium the probability of finding a sphaleron-like configuration in the hot plasma is given by the Boltzman weight of the sphaleron $\exp \left(-E_{s p} / T\right)$. At temperatures high compared to $M_{W}$ but low compared to $M_{W} / \alpha$ transitions over the barrier are governed by classical statistical mechanics and become quite rapid at $T \sim M_{W} / \alpha$ [2]. In this regime one is supposed to study time independent solution to the equation of motion. Three dimensional static gauge and Higgs fields of the sphaleron type [3] and its various deformed versions [4]. It should be noted however that fermion level crossing is not to be restricted only to solutions to the electroweak equations of motion as backgrounds to the Dirac equation. The term "sphaleron deformation" will henceforth define the general class of gauge and Higgs field sphaleron-like configurations that admit level crossing.

Eq.(1.2) provides us with the sufficient condition for level crossing to occur in the hot primordial plasma and in the presence of large thermal fluctuations of the gauge and Higgs fields. More precisely by adopting a "real time evolution" model

of a thermal fluctuation of a classical field we may consider a continuous string of static fields $\phi(x, t), A_{i}(x, t), t \in[0,1]$ that interpolates between two adjacent vacua with $t=0$ and $t=1$. (We may identify time with the Chern-Simons number and 
concentrate on the $n=0,1$ sectors of the vacuum). Provided that our set of static configurations satisfies (in the $A_{0}=0$ gauge) appropriate boundary conditions there will be a single level crossing from the lowest positive fermion eigenstate into the highest negative one. These are given by

$$
\begin{gathered}
A_{i}=0, \quad \phi=\phi_{0} \quad \text { at } t=0, \\
A_{i}=g^{-1} \partial_{i} g, \quad \phi=g \phi_{0} \quad \text { at } t=1 .
\end{gathered}
$$

Here $g$ is a large gauge transformation and $\phi_{0}$ is a constant $S U(2)$ doublet.

But at exactly which configuration and with what CS number will this occur? The issue from the phenomenological point of view may sound inconsequential and academic. Indeed in the minimal electroweak theory rapid fermion level crossing is the one condition for electroweak baryogenesis in the early Universe believed to be best understood and established. We feel nevertheless that the elucidation of subtle aspects of a well grounded effect to be always worthwhile. In this spirit we will try to answer the previously posed question. The topological condition (1.2) does not single out any specific sphaleron-like configuration. Indeed for the case of massless fermions and in the absence of Yukawa interactions numerical studies have corroborated to this conclusion [5] while explicit fermion zero mode constructions have been given only for in the specific sphaleron backgrounds with $\mathrm{CS}=1 / 2[6]$.

The case of Yukawa interactions however appears to be somewhat more subtle. The presence of a zero in the Higgs field and the fermion mass matrix appears to be an additional necessary condition intuitively for level crossing to occur. Indeed this appeared to be the case at least for the sphaleron case in a recent study [7]. Moreover one can observe in the same treatment of the problem that in the presence of a constant Higgs field the normalizability of the zero mode is lost. By analogy with the massless fermion case discussed previously it would be reasonable to expect this physical picture to hold true for a sphaleron like configuration with $\mathrm{CS} \neq 1 / 2$. Surprisingly recent numerical simulations [8] reveal that there exist sphaleron deformations with $\mathrm{CS} \neq 1 / 2$ in the presence of Yukawa interactions and a constant Higgs field throughout space, which allow for level crossing at least for small values of Yukawa couplings. In the present note we take up again the investigation of the effect of Yukawa interactions on the level crossing in the two dimensional Abelian-Higgs model.

We do it by introducing the Atiyah-Patodi-Singer (APS) index (the $\eta$ invariant) that measures the spectral asymmetry of a 3-dimensional Dirac operator in a continuous set of external static gauge + Higgs fields $A_{t}, \phi_{t}$ with $t \in\left[t_{0}, t_{1}\right]$ [9]. It is defined to be

$$
\begin{gathered}
\eta=\sum_{\lambda_{k}>0} 1-\sum_{\lambda_{k}<0} 1= \\
=\sum_{k} \frac{\operatorname{sign} \lambda_{k}}{\left|\lambda_{k}\right|^{s}} \text { at } \operatorname{Re} s>0, \quad s \rightarrow 0,
\end{gathered}
$$

where the second line gives a regularized expression for the spectral asymmetry. The symbol $\sum^{\prime}$ implies a sum over all non-zero eigenvalues $\lambda_{k}$ of the Dirac operator and 
$s$ is taken to be a complex parameter. Here the boundaries of the set of fields are not to be identified with the vacuum states with $\mathrm{CS}=0$ and 1 respectively. More geometrically they are the three dimensional connections on a manifold of a cylinder $M^{3} \times I$ with $t$ parametrizing $I=\left[t_{0}, t_{1}\right]$. The APS index theorem then [9] implies that if the eigenfunctions of a properly defined 4-dimensional $\not_{4}=\partial / \partial_{t}+\not D_{3}$ Dirac operator are to be normalizable on $M^{3} \times I$ then

$$
\eta\left(A_{t_{1}}\right)-\eta\left(A_{t_{0}}\right)=\frac{1}{16 \pi^{2}} \int_{M^{3} \times I} \operatorname{Tr} F^{2}-2 q,
$$

where $q$ stands for Pontryagin index here taken to be 1. It can be shown that when an eigenvalue of $D_{3}\left(A_{t}\right)$ crosses the zero axis at some $t=t^{*}$ the spectral asymmetry varies discontinuously by 2 units. It thus has a continuous $\left(\eta_{c}\right)$ and discontinuous parts (spectral flow $\left[t^{*}\right]$ ). The latter is twice the number of zero level crossings (see Fig.1)

$$
\eta\left(A_{t_{1}}\right)-\eta\left(A_{t_{0}}\right)=\int_{t_{0}}^{t_{1}} d t \frac{d}{d t} \eta_{c}\left[A_{t}\right]+2 \times \text { spectral flow }\left[t^{*}\right] .
$$

It can further be shown that

$$
\int_{t_{0}}^{t_{1}} d t \frac{d}{d t} \eta_{c}\left[A_{t}\right]=\frac{1}{16 \pi^{2}} \int_{M^{3} \times I} \operatorname{Tr} F^{2}
$$

We may observe two interesting limits. In the case where the boundaries $A_{0}, A_{1}$ are identical to the vacua at $\mathrm{CS}=0$ and 1 respectively we recover the usual 4-dimensional Pontryagin index relation. In the opposite limit where $t_{0} \sim t_{1} \sim t^{*}$ we have that

$$
\text { -spectral flow }=\text { topological charge } q=1 \text {. }
$$

Interestingly both the 3-dimensional spectral flow and the 4-dimensional index probe the same homotopic group $\pi_{3}(S U(2))=\mathbf{Z}$. The spectral flow across the static configuration $A_{t^{*}}$ can be computed by its parity anomaly [10, 11. In equations $\eta \sim \operatorname{Im} W(A)$. The $\eta$ invariant is proportional to the parity anomaly which is given by the imaginary part of the effective action of the 3-dimensional configuration of the gauge field.

In section 2 we illustrate the general formalism by applying it to the two dimensional Abelian-Higgs model with massless fermions and no Yukawa interactions. We demonstrate spectral flow of the $\eta$ invariant at sphaleron configurations of CS $=1 / 2$. In section 2 we investigate the effect of Yukawa interactions. We find that a constant Higgs field with no zero is compatible with the existence of a normalizable zero mode of the one dimensional Dirac operator. Interestingly this contrasts with the electroweak sphaleron case in $3+1$ dimensions about which we made some remarks before. Furthermore Yukawa interactions may induce sphaleron deformations with $\mathrm{CS} \neq 1 / 2$ which possess normalizable zero modes and hence allow for level crossing as well. We finally examine the presence of both effects in the realistic electroweak theory. 


\section{Abelian-Higgs model with massless fermions}

Here we consider an Abelian-Higgs model as a simple example which demonstrates the role of parity anomaly for the description of the level crossing phenomenon.

We consider the euclidean version of the theory whose Lagrangian reads as follows [12]

$$
L=\psi_{R}^{+} i \bar{D} \psi_{L}+\psi_{L}^{+} i D \psi_{R}
$$

Here $D=\partial-i A, \bar{D}=\bar{\partial}-i \bar{A}, \partial=\partial / \partial z, \bar{\partial}=\partial / \partial \bar{z}$, and $z=x_{1}+i x_{2}, \bar{z}=x_{1}-i x_{2}$, $A=A_{1}-i A_{2}, \bar{A}=A_{1}+i A_{2}$. The fermionic fields $\psi_{L}, \psi_{R}, \psi_{L}^{+}$, and $\psi_{R}^{+}$are chiral components of the corresponding Dirac spinors. For definiteness we fix that $x_{1}$ is "time", while $x_{2}=x$ is a (space) coordinate on a circle.

The $\eta$ invariant is a functional of a $0+1$ dimensional external gauge field. To calculate the $\eta$ invariant let us choose the Hamiltonian gauge $\left(A_{1}=0\right)$ and write down the $0+1$ dimensional Dirac operator for fermions with a definite chirality

$$
D_{x}=\partial_{x}-i A_{x}
$$

It is easy to find the spectrum of this operator. The equation for its eigenvalues $\lambda$ is given by

$$
\left(\partial_{x}-i A_{x}\right) \psi_{\lambda}=i \lambda \psi_{\lambda}
$$

We fix the anti-periodic boundary condition for the corresponding wave function on a circle. It reads as

$$
\psi_{\lambda}(x)=\exp \left(\int_{-L / 2}^{x} A_{x} d x+i \lambda x\right) \psi_{0},
$$

where $\psi_{0}$ is a non-vanishing constant. Using the anti-periodicity condition we get

$$
\lambda_{n}=-2 \pi a / L+\pi / L+2 \pi n / L
$$

where $n \in \mathbf{Z}$, and $a=\frac{1}{2 \pi} \int_{-L / 2}^{L / 2} d x A_{x}$ is the Chern-Simons functional for the $(0+1)$ case.

For definiteness we assume that $0<a<1 / 2$ so that we restrict ourselves to computing the continuous part of $\eta$. The partition function now reads [13]

$$
Z=\text { const } \times \prod_{n=-\infty}^{\infty}\left(n+\frac{1}{2}-a\right)=\cos a \pi
$$

Here we introduced a constant which does not depend on the external gauge field. It is clear that this result is not gauge invariant as it changes its sign when one makes a big gauge transformation $a \rightarrow a+1$. This is actully a result of a special choice of regularization as discussed in ref. 14. With an appropriate regularization we can still restore the gauge invariance. In this case we effectively get an additional counter term proportional to the Chern-Simons functional $a$. Simultaneously we loose the parity invariance of the theory as can be seen from the modified partition function

$$
Z=\cos \pi a e^{i a \pi}
$$


Now we can extract the imaginary part of this partition function to be identified with a parity anomaly. It is given by $\operatorname{Im} \log Z$ as follows

$$
\eta[A]=\frac{2}{\pi} \operatorname{Im} \log Z=2 a+\eta_{\text {step }},
$$

where $\eta_{\text {step }}$ is a discontinuous part of the $\eta$ invariant. This expression is gauge invariant under big gauge transformations due to the discontinuous part $\eta_{\text {step }}$. It is easy to see that the jumps of $\eta_{\text {step }}$ correspond to half integer values of the ChernSimons functional $a=n+1 / 2, n \in \mathbf{Z}$.

In what follows we present a different but equivalent way to compute the parity anomaly through the spectral asymmetry of the Dirac operator. Using the $\zeta$ regularization we have [10

$$
\eta[A]=\left.\sum_{n} \frac{\operatorname{sign} \lambda_{n}}{\left|\lambda_{n}\right|^{s}}\right|_{s \rightarrow 0} .
$$

Taking once again for definiteness $0<a<1 / 2$ we get

$$
\eta[A]=1+\sum_{n=1}^{\infty}\left(\frac{1}{|n-a+1 / 2|^{s}}-\frac{1}{|n+a-1 / 2|^{s}}\right) .
$$

To determine the dependence of this functional on $a$ we calculate its derivative

$$
\frac{\partial}{\partial a} \eta[A]=\sum_{n=1}^{\infty}\left(\frac{s}{(n-a+1 / 2)^{s+1}}+\frac{s}{(n+a-1 / 2)^{s+1}}\right) .
$$

This infinite sum diverges at $s \rightarrow 0$ and to the leading order it is given by $1 / s$. We thus get the same result for the continuous part of the parity anomaly as above. The discontinuous part obviously appears in such a calculation when we accurately take into account first terms in the sum in eq.(2.9).

Finally let us calculate the phase shift in the APS theorem for this model. Indeed we may use the Ward identity for the $\gamma_{5}$ current. We define the $\gamma_{5}$ current as follows

$$
j_{5}^{\mu}=\psi^{+} \gamma_{\mu} \gamma_{5} \psi=\psi_{R}^{+} \gamma_{\mu} \psi_{L}-\psi_{L}^{+} \gamma_{\mu} \psi_{R}
$$

where $\gamma_{1}=\sigma_{1}$ and $\gamma_{2}=\sigma_{2}$, while $\gamma_{5}=\sigma_{3}$. This current has an anomaly and the anomalous Ward identity for its divergence reads

$$
<\partial_{\mu} j_{\mu}=\frac{i}{2 \pi} \epsilon^{\mu \nu} F_{\mu \nu}+2 m \psi^{+} \gamma_{5} \psi>
$$

Integrating over $x$ and $t$ the last term in the right hand side gives the difference of left- and right- handed fermionic zero modes. Usually by assuming that the external field rapidly decreases at infinity one can easily deduce from the above equation the Atiyah-Singer index theorem. In that case the two dimensional integral of the divergence of the axial current should be zero. 
If instead we assume that the external field does not vanish this term makes a non-zero contribution to the left hand side. Let us assume that the external field rapidly decrease at large $x$, while it does not vanish as $t \rightarrow \infty$. In this case we get

$$
\frac{1}{i}<Q_{5}(t=-\infty)-Q_{5}(t=+\infty)>=\int d x \frac{1}{2 \pi} \epsilon^{\mu \nu} F_{\mu \nu}+2\left(n_{+}-n_{-}\right),
$$

where $n_{+}\left(n_{-}\right)$are the numbers of left (right)-handed fermionic zero modes with the chiral charge $Q_{5}$ being defined as follows

$$
Q_{5}=\int d x \psi^{+} \gamma_{1} \gamma_{5} \psi
$$

Let us now calculate the expectation value of $Q_{5}$ in the presence of a gauge field

$$
\begin{gathered}
<Q_{5}>=\int d x<\psi^{+} \gamma_{1} \gamma_{5} \psi>= \\
=\int d x<\operatorname{Tr}\left(G_{R L}(x, y) e^{-i \int_{y}^{x} d x_{\mu}^{\prime} A_{\mu}}-G_{L R}(x, y) e^{-i \int_{y}^{x} d x_{\mu}^{\prime} A_{\mu}}\right)>\left.\right|_{x \rightarrow y} .
\end{gathered}
$$

Here the Green functions $G_{R L}(x, y)$ and $G_{L R}(x, y)$ obey the equations

$$
i \bar{D} G_{R L}(x, y)=\delta^{2}(x-y), \quad i D G_{L R}(x, y)=\delta^{2}(x-y) .
$$

The exponentials above are introduced in order maintain the gauge invariance under regularization (point splitting). It is convenient to use the following representation for the gauge field

$$
A_{\mu}=\partial_{\mu} \alpha+\epsilon_{\mu \nu} \partial^{\nu} \beta
$$

Here $\alpha$ and $\beta$ are scalar functions. Then it is easy to check that

$$
G_{R L}(x, y)=\frac{1}{2 i \pi} e^{i(\bar{\phi}(x)-\bar{\phi}(y))} \frac{1}{\bar{z}-\bar{z}^{\prime}}, \quad G_{L R}(x, y)=\frac{1}{2 i \pi} e^{i(\phi(x)-\phi(y))} \frac{1}{z-z^{\prime}},
$$

where

$$
\bar{\phi}=\alpha+i \beta, \quad \phi=\alpha-i \beta,
$$

and $z=x_{1}+i x_{2}, z^{\prime}=y_{1}+i y_{2}$.

Taking the limit $x \rightarrow y$ we get

$$
<Q_{5}>=-\frac{i}{\pi} \int d x \partial_{1} \beta=-2 i a=-i \eta[A] .
$$

This expression is proportional to the Chern-Simons functional $a=\frac{1}{2 \pi} \int d x A_{2}=$ $\frac{1}{2 \pi} \int d x_{2}\left(\partial_{2} \alpha-\partial_{1} \beta\right)$ for periodic $\alpha$. We thus get once more the same result for the $\eta$ invariant. 


\section{Abelian-Higgs model with Yukawa interactions}

Let us now consider the level crossing phenomenon in the presence of Yukawa couplings.

The lagrangian now reads

$$
L=\psi_{R}^{+} i \bar{D} \psi_{L}+\eta_{L}^{+} i \partial \eta_{R}+i H \psi_{R}^{+} \eta_{R}+i H^{*} \eta_{L}^{+} \psi_{L}
$$

Here $H$ is a complex Higgs field which can have a non-vanishing vacuum expectation value at infinity. In this case the $U(1)$ gauge invariance is spontaneously broken. An additional neutral fermion is introduced to generate a mass of the fermion $\psi$. The lagrangian above is of course anomalous at the quantum level and therefore we shall assume that there is another charged fermion with the same charge but of an opposite chirality.

We take the same sphaleron configuration as above and the Hamiltonian gauge. The reduced $1+0$ lagrangian now reads

$$
L=-\psi_{R}^{+} D_{x} \psi_{L}+\eta_{L}^{+} \partial_{x} \eta_{R}+i H \psi_{R}^{+} \eta_{R}+i H^{*} \eta_{L}^{+} \psi_{L}
$$

We are unable to find the spectrum for this particular Hamiltonian. We observe, however, that for a given value of the $\eta$-invariant there exist a continuous set $\Omega$ of Hamiltonians that gives rise to it. Each member is related to the other by a constant matrix. On the basis of this property of the $\eta$-invariant we pick a more manageable Hamiltonian for which we can find its spectrum and determine our sought after $\eta$ invariant. Specifically our $\eta$-invariant is given by the imaginary part of the logarithm

of the fermionic determinant $(\eta \propto \operatorname{Im} \operatorname{det} \not D(A))$. We observe that the value of $\eta$ is the same if we choose $\not D^{\prime}(A)=\not D(A) \tau_{3}$, where $\tau_{3}$ is a Pauli matrix. In our particular model (eq.(3.2)) we compute the $\eta$-invariant by redefining our Dirac operator in the way indicated above. This is accomplished by effectively changing the sign of $\psi_{L}$. Our modified Dirac equation is given by

$$
\begin{aligned}
& D_{x} \psi_{L}+i H \eta_{R}=i \lambda \psi_{L}, \\
& \partial_{x} \eta_{R}-i H^{*} \psi_{L}=i \lambda \eta_{R} .
\end{aligned}
$$

The Dirac operator has the form

$$
\hat{D}=\left(\begin{array}{cc}
D_{x} & i H \\
-i H^{*} & \partial_{x}
\end{array}\right)
$$

This operator is not anti-hermitean, but its eigenvalues will be shown to be purely imaginary.

It is now convenient to remove the gauge field from the covariant derivative by a gauge rotation of the wave function

$$
\psi_{L}=g(x) \psi(x)=e^{i \int_{L / 2}^{x} d x A_{x}} \psi, \quad \eta_{R}=\eta .
$$


Now it easy to see that the (rotated) Higgs field enters as external non-abelian gauge field in the equation. However this "gauge" field is anti-hermitean. Thus the general solution to the Dirac equations reads

$$
\psi_{L}=e^{i \lambda x}\left(\begin{array}{cc}
g(x) & 0 \\
0 & 1
\end{array}\right) U(x) q_{0},
$$

where $q_{0}$ is a constant two-components wave function for $\psi_{L}$ and $\eta_{R}$ fermions, and

$$
\begin{gathered}
U(x)=P \exp \left(-\int_{-L / 2}^{x} B\right), \\
B=\left(\begin{array}{cc}
0 & i H g^{*} \\
-i H^{*} g & 0
\end{array}\right) .
\end{gathered}
$$

The matrix $B$ is hermitean, and $P$ stands for path ordering.

In order to determine the eigenvalues we should take into account the antiperiodic boundary conditions for fermions

$$
e^{-i \lambda L / 2} q_{0}=e^{i \lambda L / 2}\left(\begin{array}{cc}
g(L / 2) & 0 \\
0 & 1
\end{array}\right) U(L / 2) q_{0}
$$

It is easy to see that

$$
\operatorname{det} U(L / 2)=1
$$

since the matrix $B$ is traceless. Moreover the diagonal matrix elements of the matrix

$$
U(L / 2)=\left(\begin{array}{ll}
x & y \\
z & v
\end{array}\right)
$$

are complex conjugated

$$
x=r e^{i \theta}, \quad v=r e^{-i \theta},
$$

where $r$ and $\theta$ are real. The equation for the eigenvalues takes the form

$$
\operatorname{det}\left[e^{i \lambda L}\left(\begin{array}{ll}
g & 0 \\
0 & 1
\end{array}\right)\left(\begin{array}{ll}
x & y \\
z & v
\end{array}\right)+1\right]=0 .
$$

After some calculation we get

$$
e^{-2 i \lambda L}+e^{-i \lambda L}(v+x g)+g=0
$$

In particular for a zero mode $(\lambda=0)$ we have

$$
1+g+x^{*}+x g=0
$$

This equation fixes the value of the Chern-Simons number $a$ for which the level crossing occurs

$$
a=\frac{1}{2}+\frac{1}{2 \pi} \arctan \frac{r \sin \theta}{1+r \cos \theta}
$$


It is clear that the angle $\theta$ can have any value. We call this phenomenon, shift of $a$ from $1 / 2$, induced level crossing. In this sense level crossing is purely an effect of the presence of Yukawa interactions. This is indeed the case as in their absence level crossing can only occur for configurations with $a=1 / 2$. Clearly (3.15) implies that in their presence, it may occur elsewhere, e.g. for $a=0$. For example a zero mode can very well exist at $a=0$.

It is interesting now to make a direct calculation of the parity anomaly in the presence of Yukawa interactions. The quadratic equation above has two real solutions. Therefore there are two branches of the spectrum

$$
\lambda_{n}^{(+)}=\lambda_{0}^{(+)}+2 \pi n, \quad \lambda_{n}^{(-)}=\lambda_{0}^{(-)}+2 \pi n,
$$

where $n$ is integer, and

$$
\lambda_{0}^{( \pm)}=\frac{\pi a}{L}+\frac{\pi}{L} \pm \arccos (r \cos (\theta-\pi a)) .
$$

The eigenvalues $\lambda_{n}^{( \pm)}$are real for $|r \cos (\theta-\pi a)| \leq 1$. In this case an appropriate representation for the $\eta$-invariant is given by

$$
\eta=\sum_{n=-\infty}^{+\infty} \frac{\operatorname{sign} \lambda_{n}^{(+)}}{\left|\lambda_{n}^{(+)}\right|^{s}}+\sum_{n=-\infty}^{+\infty} \frac{\operatorname{sign} \lambda_{n}^{(-)}}{\left|\lambda_{n}^{(-)}\right|^{s}} .
$$

As we are interested in the more general case where $\lambda_{n}^{( \pm)}$are complex $(\mid r \cos (\theta-$ $\pi a) \mid>1$ ) we identify the $\eta$-invariant with the imaginary part of the logarithm of the determinant (Im $\log \operatorname{det} \hat{D}$ ) of the Dirac operator which is given by eq.(3.4). Its evaluation gives us a similar result with the massless case, namely

$$
\eta=\lambda_{0}^{(+)}+\lambda_{0}^{(-)}+\text {const. }=2 a+\text { const. }
$$

This a result agrees with our argument that the continuous part of $\eta$ invariant is essentially proportional to the Chern-Simons number and independent of the Yukawa couplings.

We illustrate level crossing induced by Yukawa as it is depicted in eq.(3.15) by a simple example. We take the Higgs field $H(x)$ to be a step function (Fig.2) so that $H=-i b_{1} \exp i 2 \pi a$ for $-L / 2<x<0$ and $H=-i b_{2} \exp i 2 \pi a$ for $0<x<L / 2$, $b_{1,2} \in \mathbf{C}$. Then the matrix $U(L / 2)$ takes the following form

$$
U(L / 2)=\exp \frac{L}{2}\left(\begin{array}{cc}
0 & b_{1} \\
b_{1}^{+} & 0
\end{array}\right) \times \exp \frac{L}{2}\left(\begin{array}{cc}
0 & b_{2} \\
b_{2}^{+} & 0
\end{array}\right) .
$$

From this equation we easily get

$$
\theta=\arg \left(\cosh \left|b_{1}\right| \cosh \left|b_{2}\right|-\sinh \left|b_{1}\right| \sinh \left|b_{2}\right| e^{i \gamma}\right),
$$

where

$$
e^{i \gamma}=\frac{b_{1} b_{2}^{+}}{\left|b_{1}\right|\left|b_{2}\right|} .
$$

It is easy to check that changing the values of the parameters $b_{1,2}$ and $\gamma$ the value of the Chern-Simons number at which the level crossing happens can be arbitrarily shifted. 


\section{Spectral flow across the electroweak sphaleron}

We can now attempt to use the concept of spectral flow across a static 3-dimensional configuration, that of the electroweak sphaleron. In the case of massless fermions we may again consider a continuous set of static gauge + Higgs fields $(\phi(x, t), A(x, t))$ parametrized by $t \in(0,1)$.

The APS index theorem (eq.(1.5)) is valid. We notice that the continuous part of the $\eta$ invariant is given by

$$
\eta_{c}\left(A_{t}\right) \propto \int_{0}^{t} d t \operatorname{Tr} F^{2} .
$$

More precisely we take in eq.(1.7) $t_{0}=0$ and $t_{1}=t$. Our experience with the 2dimensional Abelian-Higgs model suggests that if we choose an interval in $t$ that excludes a discontinuous jump for the $\eta$ invariant, i.e. consider only the behaviour of its continuous part $\eta_{c}\left(A_{t}\right)$ that should be proportional to the Chern-Simons functional for $A_{t}$ plus a constant. We now want to extend this argument to three dimensions.

We choose a gauge for the 3 -dimensional gauge fields in a $t$-interval that does not include any discontinuities for the $\eta$ invariant so that they rapidly decrease at spatial infinity. As before the $\eta_{c}\left(A_{t}\right)$ must be proportional to the Chern-Simons functional for $A_{t}$ (plus a constant). Furthermore a discontinuity in the $\eta$ inavariant across a particular sphaleron like configuration $\left(\phi_{s p}\left(x, t^{*}\right), A_{s p}\left(x, t^{*}\right)\right)$ signals similarly a level crossing, the existence of a normalizable zero energy solution, and hence fermion level crossing. As we previously stated this may occur at configurations with $\mathrm{CS} \neq 1 / 2$.

It is less trivial to extend the same argument to the case of Yukawa interactions with massive fermions. Indeed in this case the $\eta$ invariant in general depends on Yukawa couplings and Higgs fields. Numerical simulations [8] suggest surprisingly that for small Yukawa couplings there exist deformed sphalerons that allow for level crossing without the necessary presence of the zero of the Higgs field. For larger values it was found, perhaps not so surprisingly, that the situation is similar to the sphaleron case [7]. Deformed sphalerons with $\mathrm{CS} \neq 1 / 2$ admit a fermion zero mode in the presence of a zero of Higgs field. The Higgs field in three dimensions appears to have an important and subtle bearing on the level crossing phenomenon. This calls for a nontrivial generalization of our previous argument for the APS index theorem, which we do not possess at the moment. We may offer nevertheless some argument for the general behaviour of the continuous part of the spectral asymmetry that we feel holds true in this case too. More specifically we argue that it is independent of the Higgs configuration and proportional to some Chern-Simons functional. Indeed in the presence of Yukawa interactions a generalization of the Atiyah-Singer index theorem has been demonstarted [15], $2 q=\frac{1}{16 \pi^{2}} \int \operatorname{Tr} F^{2}$. We can now put it in the language of the APS index theorem (eqs.(1.5),(1.7)). Indeed from eq.(1.7) we can rewrite eq.(1.5) as follows

$$
\frac{1}{16 \pi^{2}} \int_{M^{3} \times\left[1, t_{1}\right]} \operatorname{Tr} F^{2}-\int_{M^{3} \times\left[t_{0}, 0\right]} \operatorname{Tr} F^{2}=\int_{M^{3} \times\left[t_{0}, t_{1}\right]} \operatorname{Tr} F^{2}-2 q .
$$


Here too $t_{0}$ and $t_{1}$ are the boundaries of the continuous set of gauge and Higgs fields $\left(A_{i}(x, t), \phi(x, t)\right), t \in[0,1]$. Moreover $A_{i}\left(x, t_{0,1}\right)$ and $\phi\left(x, t_{0,1}\right)$ are not the vacua with $\mathrm{CS}=0,1$ respectively. It is obvious that from eq.(4.2)

$$
\frac{1}{32 \pi^{2}} \int_{M^{3} \times\left[1, t_{1}\right]} \operatorname{Tr} F^{2}=-1+\frac{1}{32 \pi^{2}} \int_{M^{3} \times\left[0, t_{1}\right]} \operatorname{Tr} F^{2} .
$$

A direct comparison with eq.(4.1) suggests that in fact eq.(4.2) is a restatment of the APS index theorem with the $\eta_{c}\left[A_{t}\right]$ being independent of the Higgs field and Yukawa couplings. This is not to be taken of course as a demonstration that the $\eta$-functional is given by the parity anomaly in the presence of Yukawa interactions. We believe this to be true here too.

We just stated there exist deformed sphalerons with $\mathrm{CS} \neq 1 / 2$ which possess zero energy normalizable solution to the 3 -dimensional Dirac equation. In fact for small values of the Yukawa couplings the presence of a zero in the Higgs field and fermion mass matrix is unnecessary. Surprisingly this is not the case with the sphaleron configuration itself with $\mathrm{CS}=1 / 2$ in contrast with the 2-dimensional Abelian-Higgs counterpart configuration. While this paradoxical difference is obscure to us we would nevertheless like to discuss the interesting possibility that the Yukawa interactions in analogy with the 2-dimensional model induce new sphaleron deformations that allow for level crossing. This certainly presupposes a physical criterion (e.g. symmetry) that can classify and distinguish the sphaleron deformations induced by Yukawa interactions from the ones that exist in their absence and allow for level crossing. While we do not possess such an analytical tool at the moment we point out that this is a formidable task too for numerical simulations of the type that recently revealed a shift in fermion level crossing due to Yukawa interactions. To interprete such a shift as a sphaleron deformation induced purely by them one must be able to exhaustively trace all possible paths of sphaleron-like transition that interpolate between two topologically distinct vacua in the case of massless fermions and no Yukawa interactions which moreover admit zero modes at deformed sphaleron backgrounds with $\mathrm{CS} \neq 1 / 2$.

\section{Conclusions}

In the present work we explored the effect of Yukawa interactions and massive fermions on fermion level crossing in the two dimensional Abelian-Higgs model. In the massless fermion case sphalerons with $\mathrm{CS}=1 / 2$ are the only static configurations that allow for level crossing. Consequently the existence of fermion zero modes for deformed sphalerons with $\mathrm{CS} \neq 1 / 2$ we interprete as a clean signature of a sphaleron deformation induced by Yukawa interactions. In the realistic case of the electroweak sphaleron-like transitions in the hot early Universe such an interpretation is not straightforward as in the massless fermion case level crossing can occur at deformed sphaleron configuration with $\mathrm{CS} \neq 1 / 2$. Our lack of any symmetry classification for such deformations renders the interpretation of the observed shift in level crossing [8] as giving rise to novel deformed sphaleron configurations induced purely by Yukawa interactions premature. 
Our clean demonstration of such a possibility in 2 dimensions, however, also points to a non-trivial generalization of the Atiyah-Patodi-Singer (APS) index the-

orem along with the equivalence between the parity anomaly of the sphaleron and the chiral anomaly for the case of Yukawa interactions.

\section{Acknowledgments}

We are grateful to Jan Ambjørn for inspiration and suggestions. We also thank him and Gudmar Thorleifsson for communicating their numerical simulation results before publication. This research is partially supported by a NATO fund. M.A. thanks the Carlsberg Foundation for financial support.

\section{References}

[1] G.'t Hooft. Phys. Rev. Lett. 37 (1976) 8;

Phys. Rev. D 14 (1976) 3432.

[2] A.G.Cohen, D.B.Kaplan and A.E.Nelson. Ann. Rev. Nucl. Part. Sci. 43 (1993). M.E.Shaposhnikov. In "Anomalous Fermion Number Non-Conservation", Lectures at ICTP Summer School, Trieste, July 1991, CERN-TH.6304/91.

[3] N.Manton. Phys. Rev. D 28 (1983) 2019;

F.Klinkhamer and N.Manton. Phys. Rev. D 30 (1984) 2212.

[4] L.G.Yaffe. Phys. Rev. D 40 (1989) 3463.

[5] J.Ambjørn and K.Farakos. Phys. Lett. B 294 (1992) 248.

[6] J.Boguta and J.Kunz. Phys. Lett. B 154 (1985) 407.

[7] M.Axenides, A.Johansen and H.B.Nielsen, 'The level crossing phenomenon with Yukawa interactions', NBI-HE-93-46 (hep-ph/9308299).

[8] J.Ambjørn, K.Farakos, S.Hands, G.Koutsoumbas and G.Thorleifsson. Preprint NBI-HE-93-00, December 1993.

[9] M.F.Atiyah, V.Patodi and I.M.Singer. Math. Proc. Cambridge Phil. Society 77, 43 (1975).

A.J.Niemi and G.W.Semenoff. Phys. Rev. Lett. 54 (1985)873; Phys. Rev. D 30 (1984) 809 .

[10] L.Alvarez-Gaumé, S.Della Pietra and G.Moore. Ann. Phys. N.Y. 163 (1985) 288.

[11] S.Forte. Nucl. Phys. B 288 (1987) 252.

[12] A.I.Bochkarev and M.E.Shaposhnikov. Mod. Phys. Lett. A 2(1987) 991. 
[13] R.Jackiw. In "Current Algebra and Anomalous". World Scientific 1985, p.305, 350.

[14] S.Elitzur, E.Rabinovichi, Y.Frishman and A.Schwimmer. Nucl. Phys. B 273 (1986) 93.

[15] A.A.Anselm and A.A.Johansen. Nucl. Phys. B 407 (1993) 313. 


\section{Figure Captions}

Fig.1. The spectral flow as denoted by the jumps of $\eta(t)$. Wherever a Dirac eigenvalue $\lambda$ crosses zero from $\lambda>(<) 0$ levels to $\lambda<(>) 0$ levels the $\eta$-invariant jumps by $-(+) 2$ units.

Fig.2. The APS index (continuous and discontinuous) for the 2-dimensional massless chiral Abelian-Higgs model. At the sphaleron configuration with $\mathrm{CS}=1 / 2$ occurs level crossing.

Fig.3. A trial Higgs field $H(x)$ step function configuration in an 2-dimensional Abelian-Higgs model with Yukawa interactions. Induced level crossing occurs at $x=0$. 